Ann. Biol. anim. Bioch. Biophys., I973, 13 (3), 335-346.

\title{
PHYSIOLOGIE DU COL DE L'UTÉRUS DE LA BREBIS. EFFET D'UN PROGESTAGENE DE SYNTHÈSE : L'ACÉTATE DE FLUOROGESTONE
}

\author{
Françoise RAYNAUD \\ Université Paris VI, Physiologie de la Reproduction, \\ Centre national de Recherches zootechniques, I. N. R. A., \\ 78350 Jouy en Josas
}

\section{RÉSUMÉ}

La structure anatomique et histologique du col utérin de Brebis en cestrus apparaît comparable à celle décrite chez la Femme, le Macaque et la Vache. En outre, au cours de l'oestrus induit par un progestagène de synthèse (FGA) aucune modification histologique particulière n'apparaît.

Les quantités d'eau et d'acide sialique présentes dans le mucus cervical de Brebis ont été mesurées au cours de l'œstrus naturel, de l'œstrus provoqué par les progestagènes et pendant la phase lutéale.

Les teneurs en eau sont identiques au cours des deux types d'œstrus mais diminuent considé. rablement pendant la phase lutéale.

L'acide sialique existe sous deux formes dans la sécrétion cervicale : $\mathrm{N}$-acétyl et $\mathrm{N}$-glycolylneuraminique.

Les quantités totales d'acide sialique présentes dans le mucus d'œestrus naturel $(543 \mu \mathrm{g}$ \pm I23/roo mg) sont nettement inférieures à celles rapportées chez la Femme et la Vache. Le mucus cervical recueilli au cours de l'œestrus induit par le FGA est caractérisé par une baisse de la quantité d'acide sialique (3I I $\mu \mathrm{g} \pm$ I23/100 $\mathrm{mg}$ ), qui devient indétectable au cours de la phase lutéale. La signification physiologique de ces résultats est discutée.

\section{INTRODUCTION}

L'anatomie et la structure du col utérin de Mammifères ont fait l'objet de nombreux travaux, comme en témoigne la revue de HAFEZ et KANAGAwa (I972). Toutefois, ces études apparaissent fragmentaires pour la majorité des espèces à l'exception de la Femme (Krantz et PhiLIIIPS, I962).

Chez les Mammifères domestiques à insémination intra-vaginale, tels les Ovins et les Bovins, le col utérin joue cependant un rôle prépondérant : il représente en effet le premier milieu de survie des spermatozoïdes dans les voies génitales femelles. 
Au moment de l'ovulation les mucopolysaccharides complexes constituant le mucus cervical subissent des modifications de leurs propriétés physiques (DANIEL, BELL, et SANGer, I966; El-NAGGar et BAKsaï-Horváth, I97I $a$; Moghissi, I966 a), de leur composition chimique (EL-NAGGAR et BAKSAÏ-HoRváth, I97 I $b$; MoghissI, Neuhaus et Stevenson, I960; Moghissi, I 966 ), qui sont le reflet de changements de l'équilibre des stéroïdes sexuels. Ainsi les variations de la teneur en acide sialique paraissent être en relation étroite avec la progestéronémie : l'augmentation de cette hormone provoque chez la Femme et la Vache une élévation de la teneur en acide sialique du mucus cervical, qui apparaît responsable de la diminution ou de l'arrêt total de la pénétration des spermatozoïdes (GibBons, I964; GibBons et MatTnER, ig66; CARL,borg et Gemzeld, i969; CARLborg, Johansson et Gemzeli, I969). Mais actuellement les informations sont limitées à ces 2 espèces et il nous a semblé intéressant de savoir si elles pouvaient être généralisées.

C'est pourquoi, dans cette communication, parallèlement à l'étude anatomique et histologique du cervix, nous avons analysé les teneurs en eau et en acide sialique du mucus cervical de la Brebis. L'intérêt d'une telle étıde est renforcé chez cette espèce puisque la généralisation de la technique de synchronisation des chaleurs par les progestagènes peut avoir des répercussions sur la physico-chimie du mucus cervical et ainsi entraîner une réduction du transport des spermatozoïdes et, par là même, du taux de fertilité, comme l'ont suggéré QuinLIVAN et RoBinson (I967, I969).

\section{MATÉRIELS ET MÉTHODES}

Animaux

Toutes les brebis sont de race Ile-de-France et ont atteint l'âge adulte.

- Un premier lot d'animaux a été utilisé pour l'étude histologique soit au cours de l'cestrus naturel, soit après induction artificielle de l'œstrus par un progestagène de synthèse (40 $\mathrm{mg}$ de FGA administrés par éponge vaginale pendant 14 jours).

- Un deuxième lot, comprenant $\mathbf{I}_{4}$ animaux, a servi pour la détermination de la teneur en eau et en acide sialique du mucus cervical. Le mucus est collecté de la façon suivante (fig. I).

Brebis entrant en œstrus le matin
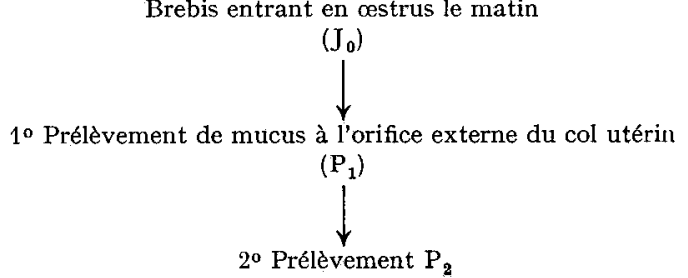

et insertion dans le vagin d'une éponge imprégnée de $40 \mathrm{mg}$ de FGA, maintenue en place 14 jours. Après retrait, injection intramusculaire

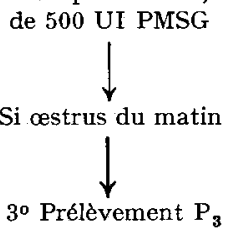

Frc. I. - Méthode de collecte du mucus cervical chez 14 brebis Ile-de-France au cours de l'cestrus naturel et au cours de l'cestrus induit par un progestagène de synthèse (FGA) 
Le premier prélèvement $\left(P_{1}\right)$ est effectué sur une femelle en oestrus le matín. L'animal est maintenu train arrière relevé; on aspire le mucus à l'orifice externe du col. Huit jours plus tard, la brebis étant considérée en phase lutéale, on pratique le même type de prélèvement $\left(\mathbf{P}_{2}\right)$. Sitôt après, on introduit au fond du vagin une éponge contenant $40 \mathrm{mg}$ de FGA qui reste en place I4 jours. Au moment du retrait, on injecte 50o UI de PMSG. Pour conserver les mêmes conditions qu'au premier prélèvement, la $3^{\mathbf{e}}$ collecte de mucus $\left(\mathrm{P}_{3}\right)$ n'est effectuée que si la femelle entre en chaleurs le matin.

La pulvérisation d'antibiotiques sur l'éponge est indispensable pour éviter un envahissement leucocytaire du mucus cervical.

\section{Hormones et produits chimiques}

Les hormones suivantes ont été utilisées : PMSG (Endopancrine), acétate de fluorogestone ou FGA ou SC-9880 (Lathévet).

Les acides $\mathrm{N}$-acétyl et $\mathrm{N}$-glycolyl-neuraminique provenaient de la Firme Sigma et Co.

\section{Méthodes histologiques}

\section{1. Étude de l'épithélium.}

Le tractus génital est fixé dans du Bouin-Hollande ou du Bouin-Allen. Après 9 jours de fixation, le col utérin est déshydraté, inclus dans la paraffine et coupé longitudinalement ou transversalement à une épaisseur de $7,5 \mu$. Divers trichromes ont été utilisés pour l'examen histologique : trichrome de Ramón y Cajal, de Mann-Dominici, ou, après réaction à l'APS (acide périodique Schiff), picro-indigo-carmin (Martoja et Martoja-Pierson, 1967).

\section{2. Étude de la musculature.}

Elle a été réalisée selon le procédé de Schmitr et Dumont (1969). Le col utérin est fixé pendant 5 heures dans un mélange à parties égales d'alcool absolu, de chloroforme et d'acide acétique cristallisable. Après élimination du fixateur par l'alcool absolu, on procède à une imprégnation des tissus par la glycérine pendant 48 heures. Après 3 lavages de I heure chacun dans l'alcool absolu, les pièces sont incluses dans la paraffine ou dans le paraplast.

L'imprégnation par la glycérine maintient parfaitement les structures et supprime presque totalement les différences de rétraction entre les divers constituants tissulaires, contrairement aux méthodes classiquement utilisées.

\section{Méthodes biochimiques}

\section{Traitement des échantillons.}

Après collecte, le mucus est pesé puis congelé à - $20^{\circ} \mathrm{C}$. Tous les prélèvements sont lyophilisés, puis pesés à nouveau. Les extraits secs sont hydrolysés par l'acide sulfurique o, $\mathrm{I}$ à $80^{\circ} \mathrm{C}$ (WARREN, 1959), pendant $45 \mathrm{mn}$, durée qui conduit à la libération maximale d'acide sialique, comme en témoigne la cinétique d'hydrolyse (fig. 2). L'acide sialique ainsi libéré est dosé selon la méthode à l'acide thiobarbiturique de WARREN (1959), en utilisant l'acide N-acétylneuraminique comme standard. La densité optique des échantillons et des témoins est mesurée à 2 longueurs d'onde $(549$ et $532 \mathrm{~nm}$ ) pour éliminer les interférences dues à d'autres saccharides libérés au cours de l'hydrolyse. La densité optique est obtenue par différence entre les mesures effectuées à 549 et $532 \mathrm{~nm}$.

\section{Identification de l'acide sialique.}

Elle a été réalisée sur une partie aliquote de chaque hydrolysat, selon un protocole décrit par Denamur et Gaye (r97I). Les sucres neutres et les osamines sont éliminés par chromatographie sur colonne d'échangeurs d'anions (Dowex $\mathrm{I} \times 8 ; 6,5 \times 0,9 \mathrm{~cm}$ ) de l'hydrolysat préalablement neutralisé. L'acide sialique adsorbé est ensuite élué par l'acide formique o,5 N. Après lyophilisation, l'éluat est repris dans un volume de $200 \mu l$ d'eau distillée et chromatographié sur papier Arches 302 dans le solvant suivant : acétate d'éthyle, acide acétique, eau (3/1/3). Après 24 heures de migration, l'acide sialique est révélé par le réactif d'Ehrlich, selon le procédé de SvENNERHor.M et SUENNERHOLM (1958). 


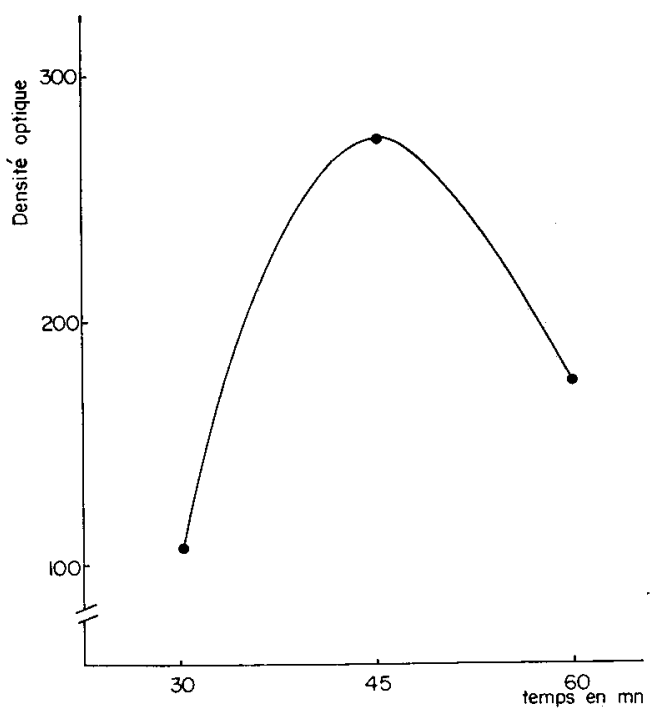

FıG. 2. - Détermination de la durèe optimale d'hydrolyse du mucus cervical de Brebis par l'acide sulfurique $0,1 \mathrm{~N}$. Cinétique d'hydrolyse

\section{RÉSULTATS}

\section{A. - Anatomie et structure du col utérin}

Comme celui des autres Mammifères, le col utérin de la Brebis est une structure à parois rigides (photo. I), de longueur moyenne : $5,19 \mathrm{~cm}$ (pour 24 animaux) avec des valeurs extrêmes de 3 et $7,7 \mathrm{~cm}$. L'analogie avec le col utérin de la Femme, (KRANTZ et PhILLIPS, I962) permet de distinguer :

- l'exocol ou exocervix, qui forme une protusion dans le vagin (fig. 3) et est revêtu d'un épithélium squameux, pluristratifié de type vaginal (photo. 2) ;

- l'endocol ou endocervix, ou canal cervical proprement dit, très sinueux, recouvert sur la majeure partie de sa longueur d'un épithélium prismatique, unistratifié de type utérin (fig. 3).

Anatomiquement, l'exocol correspond à la protusion vaginale. Si on considère l'épithélium, la délimitation entre exocol et endocol devrait être plus interne, puisque l'épithélium pluristratifié de l'exocol recouvre une faible partie du canal endocervical (fig. 3).

La muqueuse de l'endocol dessine des replis nombreux et profonds, qui s'enfoncent jusqu'à la base des muscles circulaires, comme le montre la photo 3 . Il ne s'agit en aucun cas de glandes. L'axe des replis est constitué d'un tissu conjonctif dense. Ceux-ci sont recouverts d'un épithélium unistratifié (photo. 4) renfermant des cellules ciliées et des cellules sécrétrices de mucus (photo. 5).

La sécrétion, mise en évidence par l'APS forme un véritable revêtement en bordure de l'épithélium (photo. 6).

Comme on peut le constater sur la photo. 7 , dans ces cellules caliciformes le 
noyau est rejeté à la base, tandis que la sécrétion occupe la partie apicale. Les cellules de la portion épithéliale proche de la lumière centrale semblent avoir une activité sécrétoire moins intense que celles situées au fond des replis comme en témoignent les photos 8 et 9 .

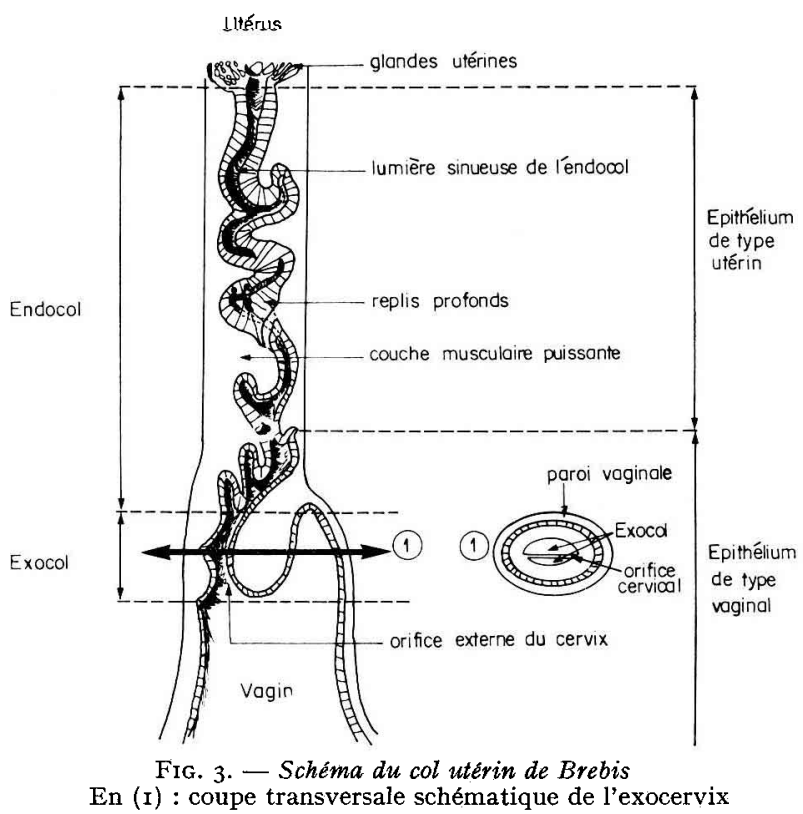

La musculature est constituée :

- d'une couche de muscles longitudinaux externes, apparaissant sous forme d'une assise discontinue dont les interstices sont occupés par du tissu conjonctif (photo Io).

- d'une épaisse couche de muscles circulaires internes, assemblés en faisceaux compacts qui s'anastomosent, enserrant un tissu conjonctif dense, comme le souligne nettement le plus fort grossissement de la photo II.

- enfin, de quelques faisceaux à orientation radiale.

\section{B. - Caractéristiques du mucus cervical}

Le mucus d'œstrus est clair, aqueux, facile à prélever, contrairement au mucus de phase lutéale, qui apparaît épais, visqueux, jaunâtre et qui, très souvent, n'affleure pas à l'orifice externe du cervix. C'est pourquoi nous n'avons pu étudier que 3 échantillons de mucus à ce stade physiologique.

Le mucus qui s'écoule après œestrus provoqué, présente apparemment toutes les caractéristiques d'un mucus d'œstrus naturel, ce qui confirme la conclusion de MOORE et al. ( 1967 ). Mais, le poids du mucus déshydraté varie significativement entre les deux prélèvements $\mathrm{P}_{1}$ et $\mathrm{P}_{3}$ (fig. 4). La quantité moyenne de mucus recueillie après œstrus induit par les progestagènes $(25 \mathrm{mg})$ est supérieure à celle collectée à l'œstrus naturel (II mg). En outre, sur les différentes collectes obtenues aux prélè- 
vements $I$ et 3 , la dispersion est plus grande pour $P_{3}$ que pour $P_{1}(2,8$ et $27,9 \mathrm{mg}$ pour $\mathrm{P}_{1} ; 5,7$ et $72,9 \mathrm{mg}$ pour $\left.\mathrm{P}_{3}\right)$. Cependant, les teneurs en eau restent semblables $\left(97,96\right.$ p. Ioo et 97,5 I p. Ioo aux I er et $3^{\mathrm{e}}$ prélèvements respectivement). En phase lutéale, la teneur en eau du mucus chute considérablement : elle ne représente que 40 p. Ioo du poids frais dans les échantillons que nous avons analysés.

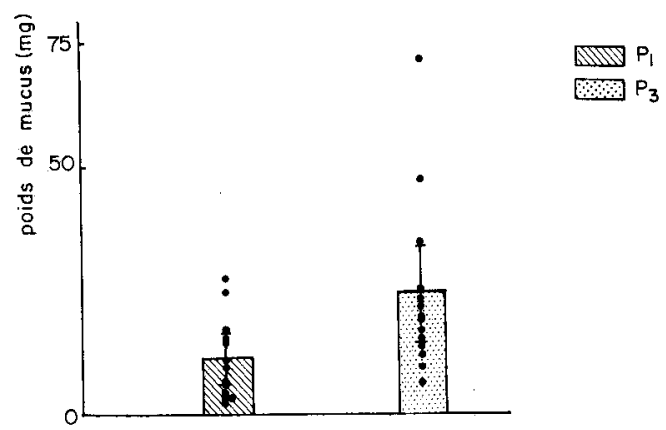

FIG. 4. - Quantité de mucus cervical collectée chez 14 brebis au cours de l'astrus naturel $\left(\mathrm{P}_{1}\right)$ et au cours de l'ostrus induit par le $F G A\left(\mathrm{P}_{3}\right)$

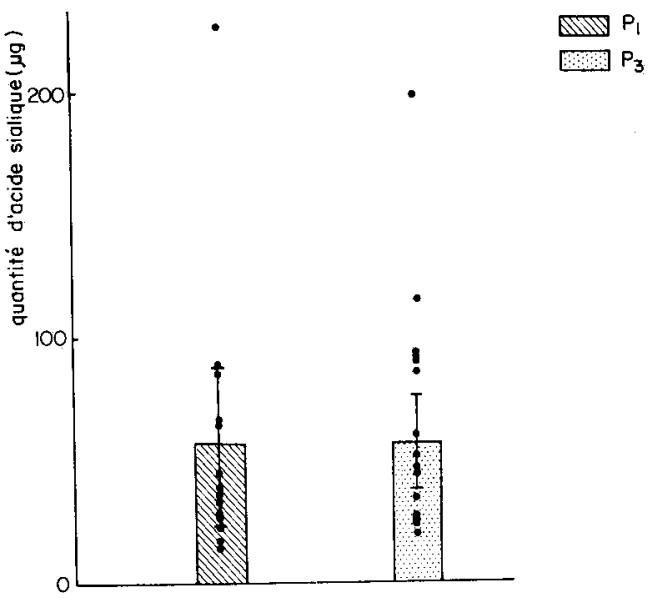

Fig. 5. - Quantité d'acide sialique (exprimée en acide $\mathrm{N}$-acétyl-neuraminique) dosée dans chaque échantillon de mucus cervical de Brebis recueilli au cours de l'cestrus naturel $\left(\mathrm{P}_{1}\right)$ ou au cours de l'ostrus induit par le FGA $\left(\mathrm{P}_{3}\right)$

C. - Détermination de la quantité d'acide sialique dans le mucus cervical

Les résultats des histogrammes consignés dans la figure 5 montrent que les quantités absolues d'acide sialique sont identiques aux prélèvements I et 3 . Comme le poids moyen du mucus augmente au $3^{e}$ prélèvement par rapport au $\mathrm{I}^{\mathrm{er}}$ (fig. 4), il en résulte que le rapport $\frac{\text { quantité d'acide sialique }}{\text { poids du mucus }}$ diminue pour $\mathrm{P}_{3}$ comparativement à $P_{1}$ (fig. 6). Les teneurs moyennes en acide sialique de ces prélèvements sont respectivement $543 \mu \mathrm{g} \pm \mathrm{I} 23 /$ roo $\mathrm{mg}$ pour $\mathrm{P}_{1}$ et $3 \mathrm{II} \mu \mathrm{g} \pm \mathrm{I} 23 / \mathrm{LOO} \mathrm{mg}$ pour $P_{3}$. Ces valeurs sont significativement différentes $(P=0,05)$. 


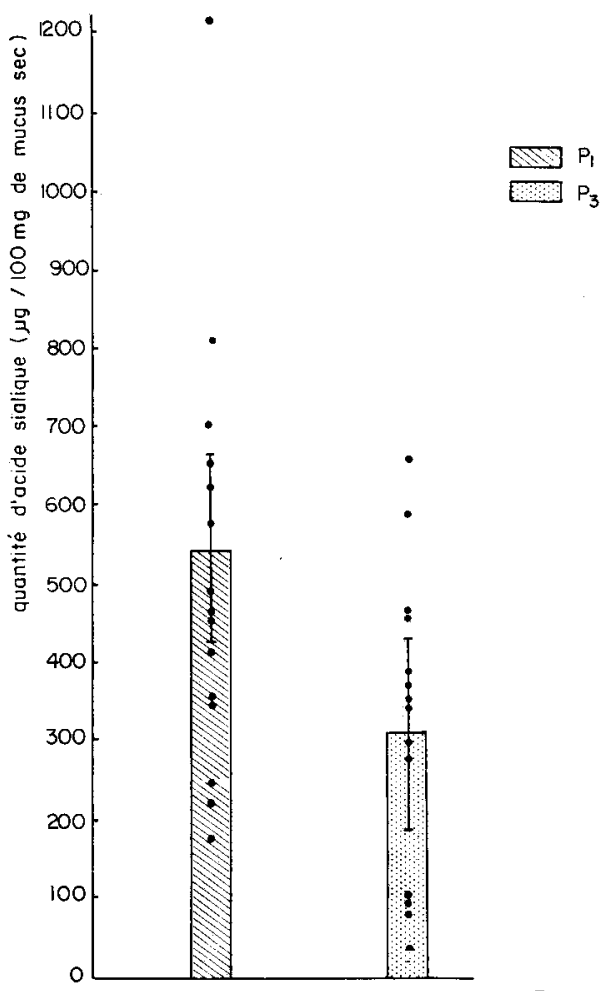

FIG. 6. - Teneur en acide sialique du mucus cervical de Brebislen cestrus naturel $\left(\mathrm{P}_{1}\right)$ ou provoqué par un progestagène de synthèse, le $F G A\left(\mathrm{P}_{\mathrm{s}}\right)$

Étude du rapport : $\frac{\text { Quantité d'acide sialique }}{\text { Poids de mucus }}$

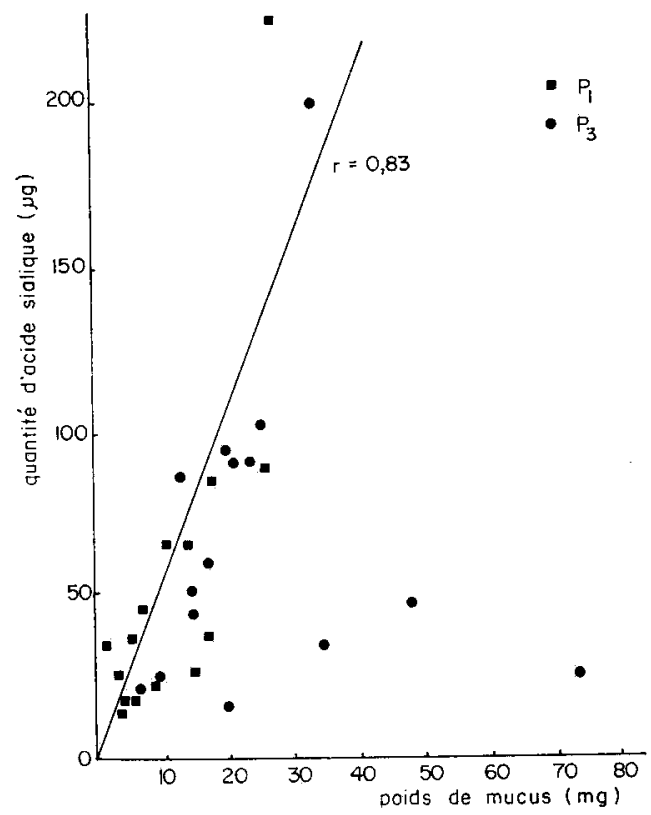

FIG. 7. - Etude de la corrélation entre la quantité d'acide sialique et le poids de mucus cervical, au cours de l'astrus naturel $\left(\mathrm{P}_{1}\right)$ ou au cours de l'cestrus synchronisé $\left(\mathrm{P}_{\mathbf{3}}\right)$ 
En outre le rapport $\frac{\text { quantité d'acide sialique }}{\text { poids du mucus }}$ apparaît plus variable après traitement par les progestagènes, puisqu'il n'existe pas de corrélation entre le poids du mucus et sa teneur en acide sialique, alors qu'il en existe une élevée à la première collecte $(r=0,83$; fig. 7$)$.

Sur les 3 échantillons qui ont pu être recueillis en phase lutéale, nous n'avons pu déceler des quantités d'acide sialique mesurables, bien que la sensibilité de la méthode de Warren permette de détecter des teneurs aussi faibles que o,or $\mu \mathrm{M}$ d'acide $\mathrm{N}$-acétylneuraminique par échantillon.

L'analyse chromatographique de l'acide sialique libéré par hydrolyse acide du mucus cervical de Brebis en œestrus naturel, puis purifié par chromatographie sur échangeur d'anions, révèle la présence de 2 composés :

- l'un possédant une mobilité analogue à l'acide $\mathrm{N}$-acétylneuraminique,

- l'autre ayant un $\mathrm{R}_{\mathrm{f}}$ comparable au dérivé $\mathrm{N}$-glycolyl.

\section{DISCUSSION}

Le col utérin de la Brebis ne présente pas de différences anatomiques et structurales notables par rapport à celui de la Femme (KRANTZ et PHILIIPS, I962) du Macaque (Cuadros, I97I) ou de la Vache (El-Banna et Hafez, I972). Chez la Brebis, la présence de callules ciliées s'accorde avec les observations effectuées à la fois chez la Femme (KRantz et Philirips, I962) et la Lapine (HafEz et Kanagawa, I972) et avec les résultats de RESTALL et LiGHTFOOT (I970) qui constatent en outre chez la Brebis une variation du nombre de ces cellules (de 30 à 60 p. Ioo) le long du col utérin.

L'existence dans le col utérin de Brebis, d'une sécrétion de mucus plus abondante dans le fond des replis que dans la lumière centrale, confirme l'hypothèse formulée par ODEBL,AD (I969) pour la Femme. Les traînées de mucus issues du fond des replis de la muqueuse, facilitent la pénétration rapide des spermatozoïdes et leur accumulation à l'extrémité aveugle de ces cryptes, comme l'ont montré MATTNER (I968) chez la Chèvre et la Vache, et MATTNER et BRADEN (I969) chez la Brebis. Ces faits pourraient également rendre compte de la faible récupération de spermatozoïdes après perfusion de l'organe $24 \mathrm{~h}$ après l'accouplement (MATrNER, I968), et $\mathrm{du}$ fonctionnement $\mathrm{du}$ cervix comme réservoir de spermatozoïdes (QUINLAN, Maré et Roux, I932, I933).

Nous devons en outre signaler qu'au cours de ce travail nous n'avons jamais pu observer une continuité entre l'épithélium superficiel et la sécrétion muqueuse qu'il élabore, même à l'extrémité aveugle des replis. On peut donc se demander si cette discontinuité ne serait pas le fait d'une rétraction supérieure du mucus cervical liée à sa nature élastique, comparativement à celle de 1'épithélium de la muqueuse.

La rigidité du col utérin de la Brebis semble moins due à une calcification, le rapport $\frac{\mathrm{Ca}}{\mathrm{P}}$ étant voisin de celui qui existe dans le muscle, qu'à une richesse particulière de l'organe en fibres élastiques (coloration de Verhoeff-Van Gieson) (en cours de vérification). 
En outre, il y a lieu de souligner qu'il n'a jamais été possible de constater de différence de structure ou d'activité sécrétoire entre les cervix d'animaux en ostrus naturel ou synchronisé.

Le mucus cervical de Brebis en phase ovulatoire apparaît, à l'instar de celui de la Femme (Pommerenke, et Rochester, r946), comme un composant à haute teneur en eau. Au cours de la phase lutéale on assiste à une chute importante de cette teneur (environ $48 \mathrm{p}$. Ioo), qui apparaît beaucoup plus accentuée que pour le mucus cervical humain, où elle n'excède pas 4 p. IOo. (PommerEnke et Rochester, 1946). Par ailleurs, le traitement par les progestagènes ne semble pas modifier la teneur en eau de la sécrétion muqueuse recueillie au cours de chaleurs induites. Ces résultats diffèrent sensiblement de ceux rapportés par SMITH (I97I) : 99,7 p. I00, dans des conditions physiologiques analogues, contre $97,5 \mathrm{I}$ p. Ioo dans cette expérience. Ces différences peuvent résulter de la méthodologie employée : ainsi, cet auteur pratique une déshydratation du mucus cervical à I0 $5^{\circ} \mathrm{C}$ pendant $24 \mathrm{~h}$ tandis que nous lyophilisons ce matériel afin d'éviter la perte des composés volatils. Néanmoins, au cours de l'œstrus synchronisé, le poids de matière sèche du mucus cervical augmente. Ainsi, l'arrêt total du traitement pourrait être responsable d'une élévation importante du niveau des hormones gonadotropes par dérépression du système hypothalamo-hypophysaire et provoquer une sécrétion œstrogénique intense, cause de l'élévation pondérale du mucus.

Dans le mucus cervical de Brebis, l'acide sialique existe sous deux formes : $\mathrm{N}$-acétyl et $\mathrm{N}$-glycolyl. On retrouve d'ailleurs ces deux composés dans le sang (SVENNERHOL,M et SVENNERHOLM, I958) et la glande mammaire de cette espèce (DENAMUR et GAYE, I97I).

Au cours de l'œstrus naturel ou synchronisé, les teneurs moyennes en acide sialique du mucus cervical, exprimées en acide $\mathrm{N}$-acétylneuraminique, apparaissent, chez la Brebis, très inférieures à celles mesurées en phase ovulatoire chez la Femme (3 p. Ioo, IAcobelili, Garcea et Angeloni, i97i ; 2 p. ioo, Carlborg, Johansson et GEMZELL, I969) et sont plus de 25 fois plus faibles que celles rapportées à la même période chez la Vache par GrbBons (I959) (I3,8 p. Ioo).

Ces différences notables des teneurs en acide sialique du mucus cervical pourraient correspondre à des variations spécifiques de la composition ou de la nature des polysaccharides. Elles ne semblent d'ailleurs pas limitées au mucus cervical, puisqu'elles apparaissent très importantes dans d'autres sécrétions et tout particulièrement dans les sécrétions salivaires (HASHIMoTo et al., I96I).

L'action de la progestérone, qui se traduit, chez la Femme et la Vache, par une augmentation notable de la teneur en acide sialique du mucus cervical, n'a pas le même effet chez la Brebis, puisqu'il ne nous a pas été possible de détecter ce composé dans les sécrétions cervicales au cours de la phase d'activité maximum du corps jaune. De plus, après traitement par les progestagènes, lors de 1'œstrus induit, la quantité d'acide sialique est significativement plus faible qu'au cours de l'œstrus normal. Ainsi, chez la Brebis, la réduction du transport des spermatozoìdes pouvant aboutir à une diminution du taux de fécondation (MOORE et al., I967; WISHAR'r, I967) ne peut s'expliquer par la présence d'une haute teneur en acide sialique modifiant le degré de réticulation des chaînes glycoprotéiques.

Reçu pour publication en mars 1973. 


\section{REMERCIEMENTS}

Nous remercions M. C. Thibault, professeur à l'Université Paris. VI, qui fut à l'origine de ce travail, M. O. Schmit de la Station de Recherche sur la Viande, pour les conseils qu'il nous a prodigués, ainsi que Mlle Aline SoLARI, qui a bien voulu assurer l'interprétation statistique des résultats. Nous remercions également, les animaliers et l'ensemble du personnel des abattoirs, sans l'aide de qui cette étude n'aurait pu être menée à bien.

\section{SUMMARY}

\section{PHYSIOLOGY OF THE UTERINE CERVIX OF THE EWE}

The anatomy and histology of the cervix are compared at estrus in normal cycling ewes and in ewes treated with intravaginal sponges impregnated with synthetic progestogen (FGA). There is no difference between the cervices of normal or synchronized ewes and the cervical structure is very similar to that reported in other species. It is possible to distinguish two structures, the outer cervix and the inner cervix, as in human and Rhesus monkey. Microscopically, the inner cervix is covered with a stratified epithelium several layers thick. The epithelium of this cervix presentsdeep folds. The monolayered epithelial cells are tall, columnar and actively secrete PAS-positive material. The squamo-columnar junction is found to be within the endocervical canal.

The changes in water and sialic acid concentration in the cervical mucus of the ewe are also studied at natural and progestogen-induced estrus and during the luteal phase.

No difference is found in water content of the cervical secretion collected at natural or induced estrus, but an important decrease is observed during the luteal phase.

Paper chromatography reveals that sialic acid is present as $\mathrm{N}$-acetyl and $\mathrm{N}$-glycolyl compounds in the cervical secretion.

The total sialic acid content of natural estrus mucus $(543 \mu \mathrm{g} \pm 123 / \mathrm{I00} \mathrm{mg})$ is much lower than that reported for woman and cow. Synchronized estrus mucus is characterized by a decrease in sialic acid concentration (3r I $\mu \mathrm{g} \pm \mathrm{I} 23 / \mathrm{I} 00 \mathrm{mg}$ ). During the luteal phase sialic acid becomes undetectable.

It is suggested that the poor sperm transport and decrease of fertilization rate observed in this species after synchronization of estrus by progestogens is not due to the presence of a high content of sialic acid, which might increase cross-linkages between the long threads of glycoprotein.

\section{RÉFÉRENCES BIBLIOGRAPHIQUES}

Carlborg L., Gemzell C., I969. Sialic acid content and sperm receptivity of cervical mucus in relation to estrogen excretion following administration of FSH. Acta. Endocrinol., 62, 7 I I-720.

Carlaorg L., Johansson D. B., Gemzel. C., 1969. Sialic acid content and sperm penetration of cervical mucus in relation to total urinary œestrogen excretion and plasma progesterone levels in ovulatory women. Acta. Endocrinol. 62, 721-731.

Cuadros A., r97r. New findings relating to the gross and microscopic morphology of the uterine cervix in the Rhesus monkey. Fertil. Steril., 22, I38-143.

DANiel D. L., Bell D. S., SANGer V. L., I966. Arborization patterns of cervico-vaginal mucus of sheep. Ohio Agric. Res. Develop. Center, Wooster, Ohio. Research Circular 146.

Denamur R. E., GAYe P. J. B., I97x. Isolement de 4 UDP-trisaccharides dans la glande mammaire et le colostrum de Brebis. Eur. J. Biochem., 19, 23-35.

El-Banna A. A., Hafez E. S. E., 1972. The uterine cervix in mammals. Am. J. Obst. Gynec., 112, $I 45-I 64$.

El-Naggar M. A., Baksal-Horváth E, I97I $a$. The physical properties of the cervico-vaginal mucus of hungarian cows during the oestrous cycle. Acta Vet. Acad. Sci. Hung., 21, 21-29. 
EL-NAGgar M. A., BAKSAÏ-HoRváth E., I97r $b$. The sugar content of the cervico-vaginal mucus of cattle during the sexual cycle, with special reference to fructose. Acta Vet. Acad. Sci. Hung., 21, I5-20.

Grbbons R. A., I959. Chemical properties of two mucoïds from bovine cervical mucus. Biochem. J.73, 209-217.

Grbbons R. A., I964. Le mucus cervical : ses propriétés physiques et chimiques et quelques observations sur le comportement des spermatozoïdes dans la sécrétion. In : Les fonctions du col utérin, 57-67, Coll. Soc. Nat. Etudes Stéril. Fécond., Masson Éd.

Gibbons R. A., Mattner P. E., I966. Some aspects of the chemistry of cervical mucus. Int. J. Fertil., 11, 366-372.

Hafez E. S. E., Kanagawa H., I972. Ciliated epithelium in the uterine cervix of the macaque and rabbit. J. Reprod. Fertil., 28, 91-94.

Hashimoto Y., Tsuiki S., Quintarelli G., Pigman W., I96r. A comparison of submaxillary glands of humans, cattle, dogs and rats. Bioch. Biophys. Acta, 48, 404-406.

I Acobelli S., Garcea N., Angeloni C., I97I. Biochemistry of cervical mucus : a comparative analysis of the secretion from preovulatory, postovulatory and pregnancy periods. Fertli. Steril., 22, 727-734.

Krantz K. E., Phillips W. P., 1962. Anatomy of the human uterine cervix gross and microscopic. Ann. N. Y. Acad. Sci., 97, 551-563.

Martoja R., MartojA-Pierson M., 1967. Initiation aux techniques de l'histologie animale. Masson Éd.

Mattner P. E., I968. The distribution of spermatozoa and leucocytes in the female genital tract in goats and cattle. J. Reprod. Fertil., 17, 253-26r.

Mattner P. E., Braden A. W. H., I969. Comparison of the distribution of motile and immotile spermatozoa in the ovine cervix. Austr. J. Biol. Sci., 22, 1069-1070.

Moghissi K. S., $1966 a$. Cyclic changes of cervical mucus in normal and progestin treated women. Fertil. Steril., 17, 663-675.

MoGHISsI K. S., 1966 b. Changes of cervical mucus proteins. Int. J. Fertil., 11, 284-286.

Moghissi K. S., Neuhaus O. W., Stevenson C. S., r960. Composition and properties of human cervical mucus. I. Electrophoretic separation and identification of proteins. J. Clin. Invest., 39, 1358I363.

Moore N. W., Quinlivan T. D., Robinson T. J., Smith J. F., r967. The fertilization of ova following natural mating or artificial insemination after withdrawal of $\mathrm{SC}-9880$ impregnated intravaginal ponges from cyclic Merino ewes. In : The control of the ovarian cycle, I69-I76, Ed. by Robinson T. J., Sydney Univ. Press.

Odeblad E., 1969. Types of human cervical secretion. Acta Europ. Fertil., 1, 99-II6.

Pommerenke W. T., Rochester N. Y., I946. Cyclic changes in the physical and chemical properties of cervical mucus. Am. J. Obst. Gynec., 52, 1023-1031.

QUinlan J., MaRE G. S., Roux L. L., r932. The vitality of the spermatozoon in the genital tract of the Merino ewe, with special reference to its practical application in breeding. Rep. Div. Vet. Serv. Anim. Ind. S. Afr., 18, 831-87o.

Quinlan J., Mare G. S., Roux L. L., I933. A study of the duration of motility of spermatozoa in the different divisions of the reproductive tract of the Merino ewe. Onderstepoort $J$. Vet. Sci., 1 , I35-I 45 .

Quinlivan T. D., Robinson T. J., 1967. The number of spermatozoa in the fallopian tubes of ewes at intervals after artificial insemination following withdrawal of SC-9880 impregnated intravaginal sponges. In : The control of the ovarian cycle, 177-194, Ed. by Robinson T. J., Sydney Univ. Press.

Quinlivan T. D., Robinson T. J., I969. Numbers of spermatozoa in the genital tract after artificial insemination of progestagen treated ewes. $J$. Reprod. Fertil., 19, 73-86.

Restall B. J., Lightfoot R. J., I970. The distribution of ciliated cells in the cervix of the ewe. A ustr. J. Biol. Sci., 23, 1265-1269.

Schmit O., Dumont B. L., I969. Méthodes d'analyse de la structure musculaire. Ann. Biol.anim. Bioch. Biophys., 8, r23-134.

SмIтн J. F., I97I. Studies on ovine infertility in agricultural regions of western Australia. Cervical mucus production by fertile and infetile ewes. Austral. J. Agric. Res., 22, 513-519.

SVennerholm E., Svennerholm L., I958. Quantitative paper partition chromatography of sialic acids. Nature, 181, II54-II55.

WARREN L., 1959. The thiobarbituric acid assay of sialic acids. J. Biol. Chem., 284, 197I-I975. 


\title{
ABREVIATIONS UTILISEES SUR LES PHOTOS
}

\author{
$\mathrm{A}=$ anastosomes \\ $\mathrm{CC}=$ cellules ciliées \\ $\mathrm{CS}=$ cellules sécrétrices \\ $\mathrm{CU}=$ col utérin \\ $\mathbf{M C}=$ assise de muscles circulaires \\ $M L=$ assise de muscles longitudinaux \\ $\mathrm{N}=$ noyau \\ $\mathrm{SM}=$ sécrétion muqueuse
}

\section{PLANCHE I}

\section{Phото I}

Aspect morphologique du tractus génital de la Brebis observé en face ventrale.

\section{Рното 2}

Coupe longitudinale dans l'exocervix : 1'épithélium qui borde 1a lumière est squameux, pluristratifié, de type vaginal ( $x$ Ioo) 

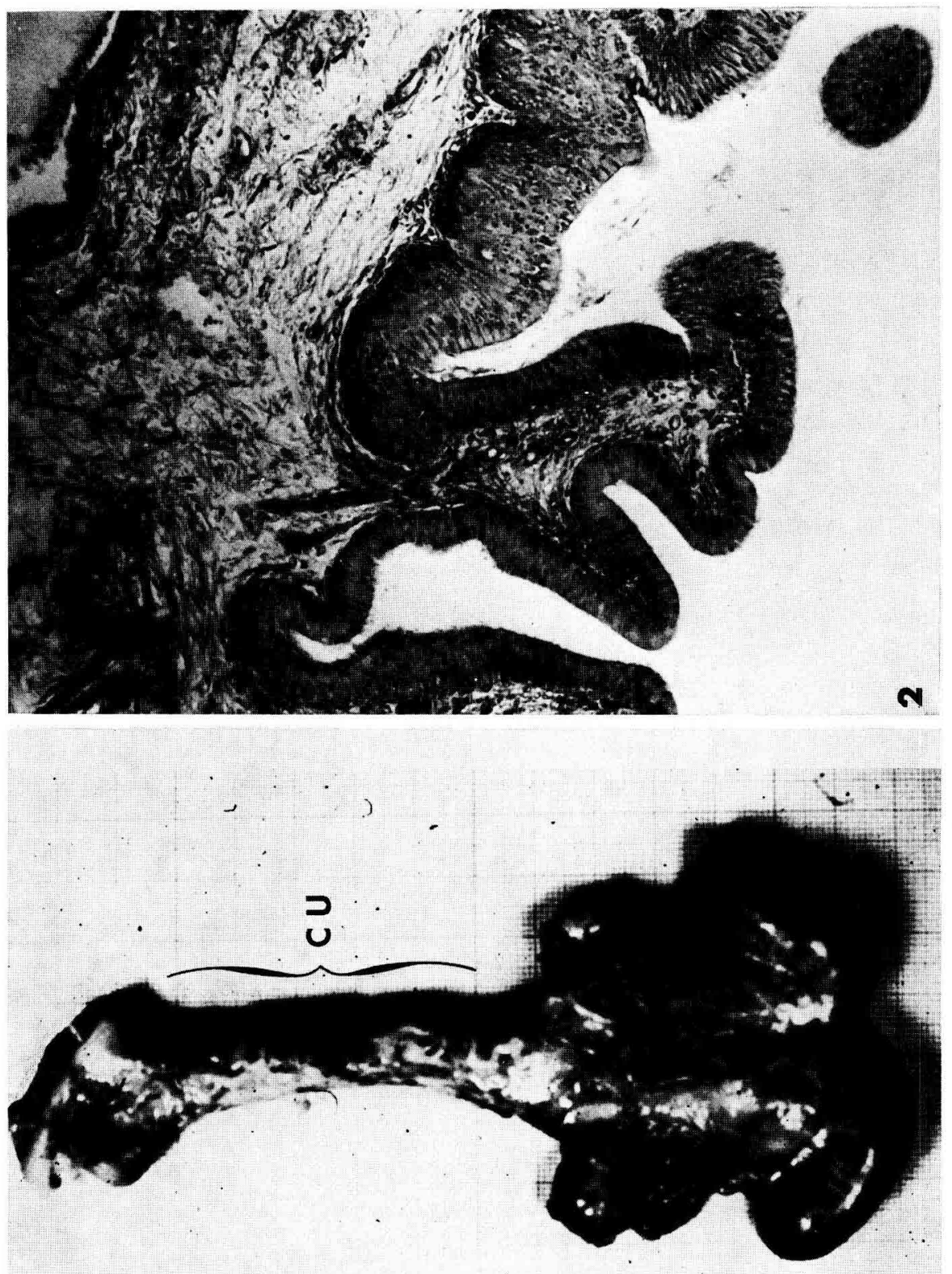

Françoise RAYNAUD 


\section{PLANCHE II}

\section{Photo 3}

Coupe longitudinale dans l'endocervix. Les replis de la muqueuse s'enfoncent jusqu'à la base des museles circulaires ( $\times 25)$.

\section{Photo 4}

L'êpithẻlium unistratifie de la muqueuse de l'endocol borde un tissu conjonctif dense $(\times 100)$.

\section{Photo 5}

Détail de cet épithêlium, montrant deux catégories cellulaires : cellules ciliées et cellules sêcrétrices $(\times 250)$. 

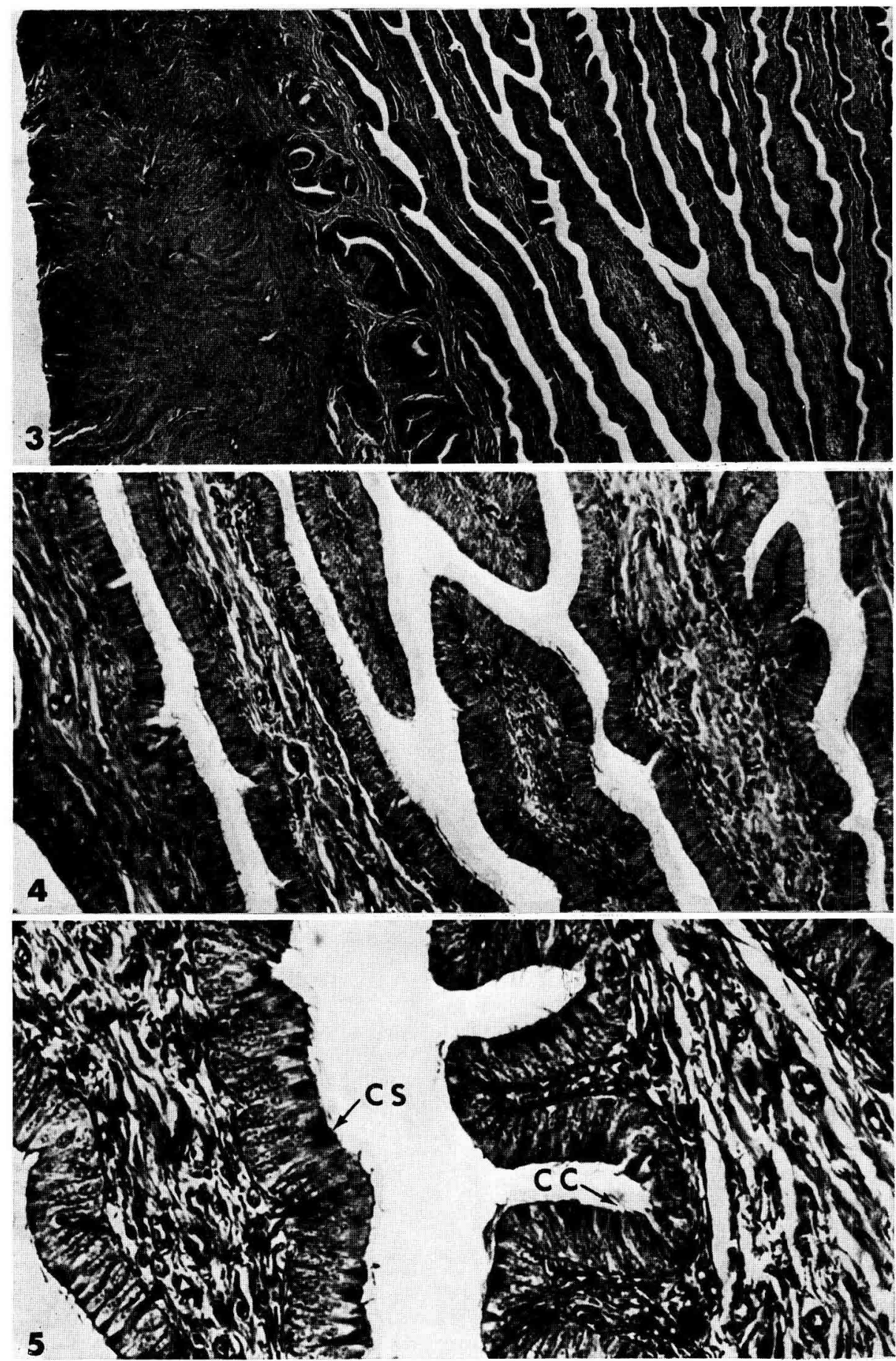

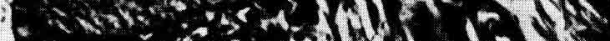

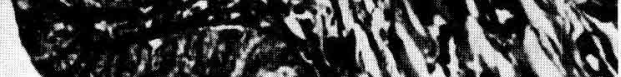

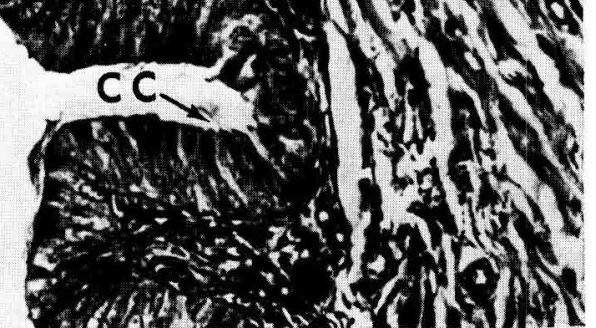




\section{PLANCHE III}

\section{Photo 6}

Coupe longitudinale dans l'endocervix La sécrêtion muqueuse de l'épithélium est míse en evidence par la réaction histochimique a l'APS $(\times 25)$.

\section{Photo 7}

Détail montrant la disposition et limportance relative du noyau et de la sécrétion muqueuse dans les cellules épithéliales ( $x 400$ ). 

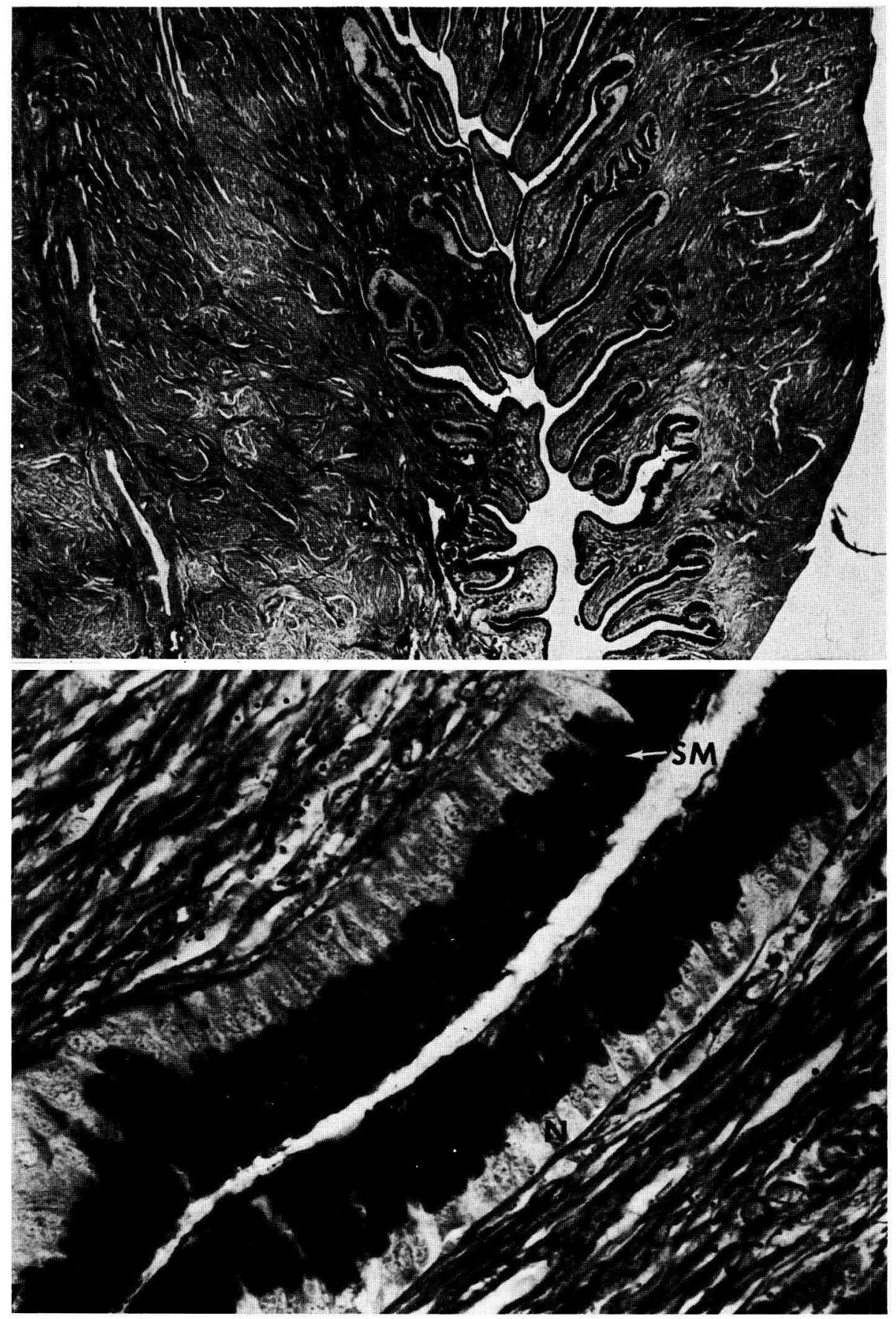


\section{PLANCHE IV}

\section{Photo 8}

La sécrétion est faible en bordure de la lumière centrale $(\times 100)$.

\section{Photo 9}

Sécrétion intense au fond des replis $(\times 100)$. 


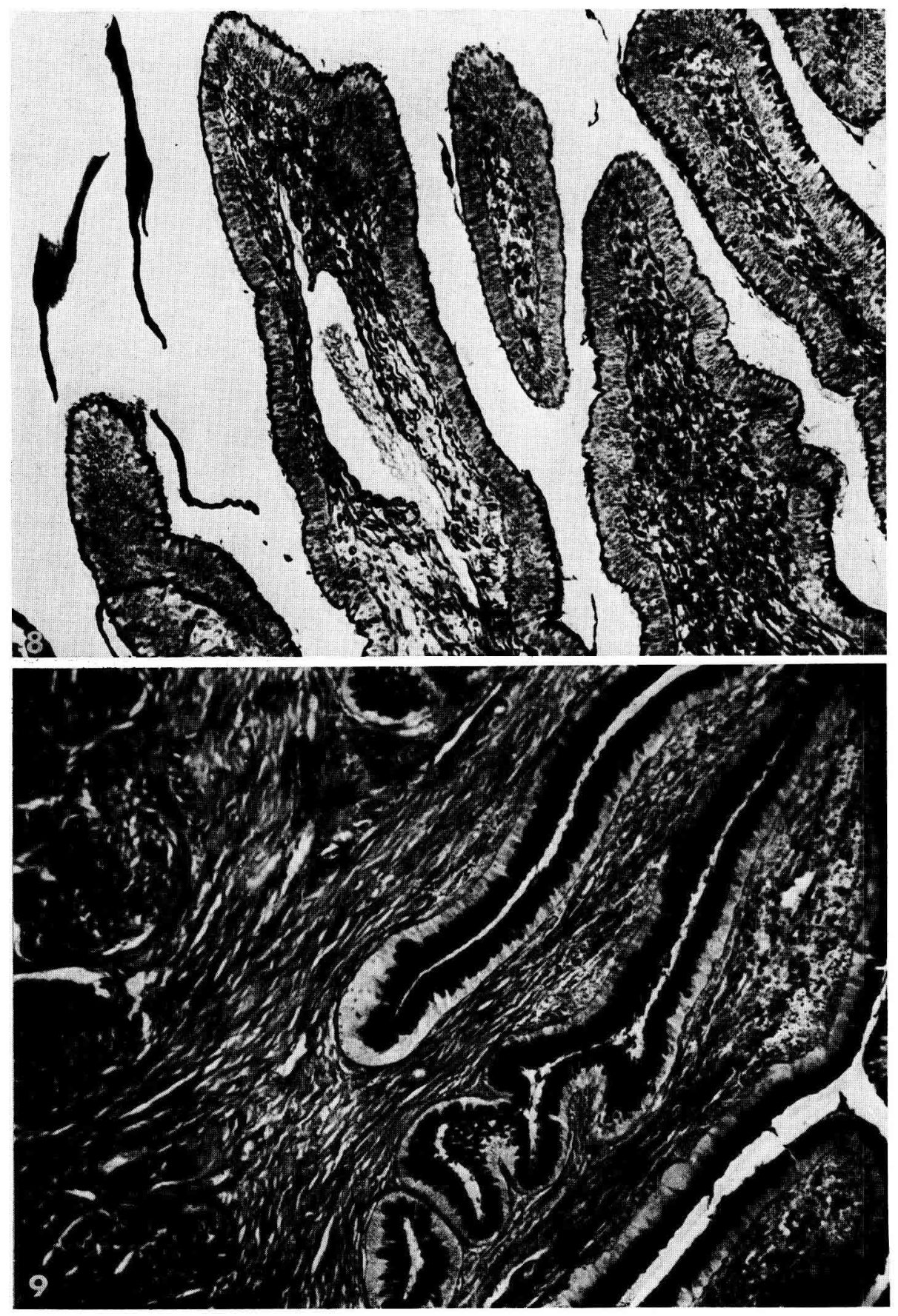




\section{PLANCHE V}

\section{Photo 10}

Coupe transversale du col utérîn de Brebis, La musculature, située sur la gauche du cliché, comporte deux assises nettement visibles: une eouche de muscles longitudinanx externes et une couche de muscles circulaires internes.

\section{Photo 11}

Détail de l'assise de muscles circulaîres, montrant les anastosomes entre les faisceaux de fibres. 


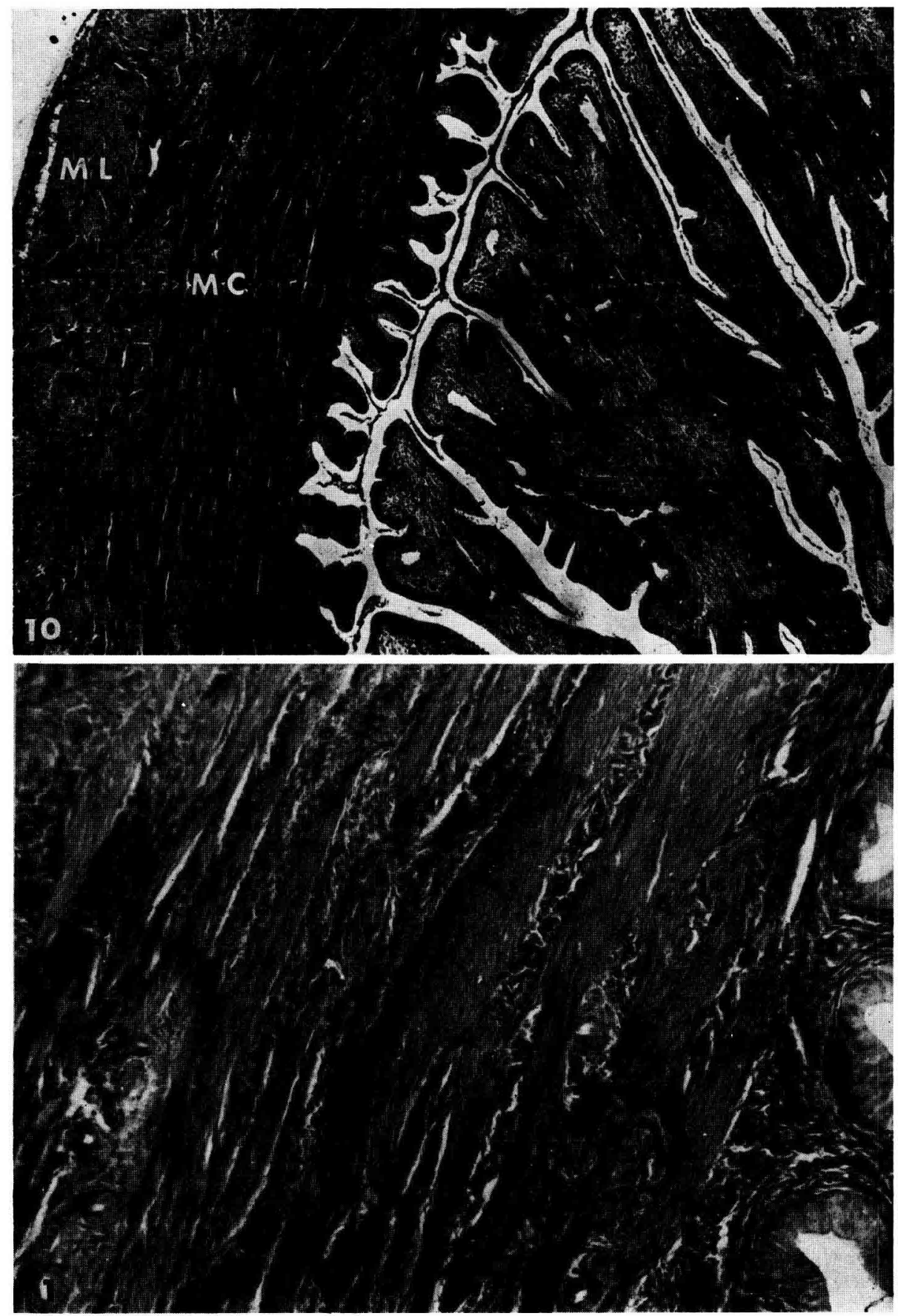

